\title{
CONTINUOUS STOLZ EXTENSIONS AND BOUNDARY FUNCTIONS $\left(^{1}\right)$
}

BY

\author{
LARRY E. SNYDER(2)
}

Introduction. This paper is concerned with properties of the boundary limit function $f$, defined on the $x$-axis, of a function $\Phi$ defined in the upper half-plane. For $\Phi$ arbitrary, except that each point $x$ on the $x$-axis is the vertex of a Stolz angle $S_{x}$ in which the limit of $\Phi$ at $x$ exists, the Stolz angle limit function $f$ is always of Baire class 2 but not necessarily of Baire class 1. However, under quite general conditions on the collection $\left\{S_{x}\right\}$ of Stolz angles used in taking the limits, the function $f$ is always of Baire class 1 .

On the other hand, given any $f$ of Baire class 1 , defined on the $x$-axis, there is a continuous function $\Phi$, defined in the upper half-plane, such that $\Phi$ converges to $f$, where at each $x$ the limit exists in every Stolz angle with vertex at $x$. Also, a number of extensions and variants of these results are given. In particular, $n$-dimensional problems as well as boundary limits along arcs will be considered.

Let $R, R^{+}$, and $R^{n}$ respectively denote the real numbers, the positive real numbers, and euclidean $n$-space. For $f: R^{n} \rightarrow R$ and $N$ an open set in $R^{n}$, let $\delta(f, N)=\sup \{|f(u)-f(v)|: u, v \in N\}$. The oscillation of $f$ at $x \in R^{n}$ is $\omega(f, x)$ $=\inf \{\delta(f, N): N$ is a neighborhood of $x\}$.

2. Stolz angle limit functions. By a Stolz angle we mean an angular sector which lies in the upper half-plane with its vertex on the $x$-axis and which meets the $x$-axis only at its vertex. A function $f: R \rightarrow R$ will be called a Stolz angle limit function for a function $\Phi: R \times R^{+} \rightarrow R$ if there is a collection of Stolz angles $\left\{S_{x}: x \in R\right\}$ such that, for each $x$ in $R, S_{x}$ has vertex $(x, 0)$ and $\Phi(p) \rightarrow f(x)$ as $p$ approaches $(x, 0)$ from within $S_{x}$.

For $x \in R$ and $0<\theta<\pi$, let $l(x, \theta)$ denote the line in the plane through $(x, 0)$ with angle $\theta$ between the $x$-axis and the line. A collection of Stolz angles is said to be uniformly directed if there is a fixed $\theta_{0}$ such that, for every Stolz angle $S$ in the collection, $l\left(x, \theta_{0}\right)$ meets $S$ if $(x, 0)$ is the vertex of $S$. In this section we consider Stolz angle limit functions obtained by limits relative to a uniformly directed collection of Stolz angles.

Presented to the Society, April 29, 1963 under the title On functions of Baire class one; received by the editors June 1, 1964, and, in revised form, October 22, 1964.

(1) This is a portion of the author's doctoral dissertation at Purdue University.

(2) Supported in part by NSF Grant No. 18920. 
THEOREM 1. If $f: R \rightarrow R$ is a Stolz angle limit function for a function $\Phi: R \times R^{+} \rightarrow R$, and if the collection $\left\{S_{x}: x \in R\right\}$ of Stolz angles, relative to which the limits are taken, is uniformly directed, then $f$ is in the first Baire class.

Proof. For the proof we will show that $f \mid P$ has a point of continuity for any nonempty perfect set $P \subset R$. From this and Baire's theorem (see [3]), it follows that the function $f$ is in the first Baire class.

Suppose there is a nonempty perfect set $P \subset R$ for which $f \mid P$ has no point of continuity. For each $n=1,2, \cdots$, let $D_{n}=\{x \in P: \omega(f \mid P, x) \geqq 1 / n\}$. It follows that $P=\bigcup_{n=1}^{\infty} D_{n}$. Since $P$ is of the second category in itself, there is an open interval $J$ and an integer $n_{0}$ such that $J \cap P \neq \varnothing$ and $D_{n_{0}}$ is dense in $J \cap P$. Since the oscillation function is upper semi-continuous, each $D_{n}$ is closed, and hence $D_{n_{0}} \supset J \cap P$.

Let $Q=$ closure $[J \cap P] . Q$ is a perfect set, and $f \mid Q$, which is identical with $f \mid P$ on $J$, has oscillation greater than or equal to $1 / n_{0}$ at each point of $J \cap P$.

Let $S_{x n}=\left\{p \in S_{x}: p=(u, v)\right.$ and $\left.v \leqq 1 / n\right\}$, and choose $M$ so that $M>16 n_{0}$. Define $A_{n}=\left\{x \in Q: p \in S_{x n} \Rightarrow|\Phi(p)-f(x)|<1 / M\right\}$. It follows that $Q=\bigcup_{n=1}^{\infty} A_{n}$ and $A_{n} \subset A_{n+1}$. Since $Q$ is of the second category in itself, there is an integer $k$ and an open interval $I \subset J$, with $I \cap Q \neq \varnothing$, such that $A_{k}$ is dense in $I \cap Q$.

Let $z$ be a fixed element of $I \cap Q$, and let $n(z)$ be the smallest positive integer $n$ such that $z \in A_{n}$. We assert that there is an open interval $V \subset I$ with $z \in V$ such that $S_{x j} \cap S_{z j} \neq \varnothing$ for any $x \in A_{k} \cap V$ and for $j=\max \{n(Z), k\}$. There is an angle $\theta_{0}$ for which $l\left(x, \theta_{0}\right)$ meets the interior of $S_{x}$ for every $x \in R$. Let the interval $(a, b)$ be the result when $S_{z j}$ is projected in a direction parallel to $l\left(z, \theta_{0}\right)$ onto the $x$-axis. The set $V=(a, b) \cap I$ possesses the required property.

Suppose that $\left\{x_{i}\right\}$ is any sequence of points in $Q$ with $z$ as limit. There is a positive integer $N$ such that $i>N$ implies $x_{i} \in V$. Let $i$ greater than $N$ be fixed and let $n$ be a positive integer such that $x_{i} \in A_{n}$. Since $A_{k}$ is dense in $V \cap Q$, there is a $w \in A_{k} \cap V$ such that $S_{w k} \cap S_{x_{i} n} \neq \varnothing$. If $\xi \in S_{z j} \cap S_{w,}$ and $\eta \in S_{w k} \cap S_{x_{i} n}$, we have

$$
\begin{aligned}
\left|f(z)-f\left(x_{i}\right)\right| \leqq & |f(z)-\Phi(\xi)|+|\Phi(\xi)-f(w)| \\
& \quad+|f(w)-\Phi(\eta)|+\left|\Phi(\eta)-f\left(x_{i}\right)\right| \leqq \frac{4}{M}<\frac{1}{4 n_{0}}
\end{aligned}
$$

However, this implies that $\omega(f \mid P, z)<1 / n_{0}$. This is a contradiction, and hence $f$ is in the first Baire class.

REMARK. Theorem 1 remains valid if the limit of $\Phi$ is only assumed to exist relative to two simple arcs which lie in the upper half-plane except for their common end-point $(x, 0)$ and which are separated by $l\left(x, \theta_{0}\right)$ where $\theta_{0}$ is independent of $x$.

The following example shows that if the Stolz angles are not restricted, the Stolz angle limit function may not be in the first Baire class. 
EXAmple 1. Let $B$ be the relative complement of the Cantor set $C$ in $[0,1]$. $B$ is the union of a countable collection of disjoint open intervals, say $B=\bigcup_{n=1}^{\infty}\left(a_{n}, b_{n}\right)$. For each $n=1,2, \cdots$, construct in the upper half-plane an equilateral triangle with the interval $\left[a_{n}, b_{n}\right]$ as its base. Define a function $\Phi$ in the upper half-plane by letting $\Phi$ have the value 1 at each point which lies in the interior of one of the triangles constructed above and letting $\Phi$ have the value 0 elsewhere.

If $x=a_{n}$ or $x=b_{n}$ for some $n$, let $S_{x}$ be a Stolz angle lying in the equilateral triangle associated with the interval $\left(a_{n}, b_{n}\right)$ and having vertex $(x, 0)$. If $x$ is any other point, let $S_{x}$ be the Stolz angle whose sides are $l(x, 5 \pi / 12)$ and $l(x, 7 \pi / 12)$. It is easily seen that the Stolz angle limit of $\Phi$ exists for this collection of Stolz angles, and the Stolz angle limit function is the function defined by

$$
f(x)=\left\{\begin{array}{l}
0 \text { if } x \geqq 1 \text { or } x \leqq 0 \\
1 \text { if } x \in\left[a_{n}, b_{n}\right] \text { for some } n, \\
0 \text { if } x \in[0,1] \sim \bigcup_{n=1}^{\infty}\left[a_{n}, b_{n}\right] .
\end{array}\right.
$$

However, $f \mid C$ has no point of continuity, so $f$ is not in the first Baire class. We note that $f$ is in the second Baire class.

We will return to arbitrary Stolz angle limit functions in $\S 4$.

3. Varying directions. Some restriction on the Stolz angles is necessary in order to have the Stolz angle limit function in the first Baire class. In this section we consider less stringent conditions than those of Theorem 1.

Definition. Let $\left\{S_{x}: x \in R\right\}$ be a collection of Stolz angles such that $S_{x}$ has vertex $(x, 0)$ for each $x$. If there is a function $\theta: R \rightarrow(0, \pi)$, with $\theta$ in the first Baire class, such that Int. $\left(S_{x}\right) \cap l(x, \theta(x)) \neq \varnothing$ for each $x$, then the class of Stolz angles is said to be $B$-directed.

THEOREM 2. If $f: R \rightarrow R$ is a Stolz angle limit function for $\Phi: R \times R^{+} \rightarrow R$, and if the collection of Stolz angles (relative to which the limits are taken) is $B$-directed, then $f$ is in the first Baire class.

Proof. Suppose there is a perfect set $P$ for which $f \mid P$ has no points of continuity. Let $D_{n}, D_{n_{0}}, Q, S_{n x}, M$, and $A_{n}$ be as in the proof of Theorem 1 .

Let $\theta: R \rightarrow(0, \pi)$ be a function in the first Baire class which "directs" the given collection on Stolz angles. For each $x \in R$, let $\alpha(x)$ be the angle between the $x$-axis and the left side of $S_{x}$ and let $\beta(x)$ be the angle between the $x$-axis and the right side of $S_{x}$. For each pair of positive integers $(n, j)$ define $A_{n j}$ to be the set of points $x$ in $A_{n}$ for which $\alpha(x)-\theta(x) \geqq 1 / j$ and $\theta(x)-\beta(x) \geqq 1 / j$. If follows that $Q=\bigcup_{n=1}^{\infty} \bigcup_{j=1}^{\infty} A_{n j}$ and $A_{n j} \subset A_{n j+1}$. Since $Q$ is of the second category in itself, there is a pair of integers $(m, k)$ and an open interval $I$ such that $A_{m k}$ is dense in $Q \cap I \neq \varnothing$. 
The function $\theta$ is in the first Baire class, so $\theta \mid Q$ has a point of continuity in $Q \cap I$. Let $z$ be such a point, and let $n(z)$ be the smallest positive integer $n$ such that $z \in A_{n}$, and let $j(z)$ be the smallest positive integer $j$ such that $z \in A_{n(z) j}$. Set $\varepsilon=4^{-1} \min \{1 / j(z), 1 / k\}$. There is an open interval $U$ containing $z$ such that if $x \in Q \cap I \cap U$, then $|\theta(z)-\theta(x)|<\varepsilon$.

Let $r=\max \{n(z), m\}$. The projection of $S_{z r}$ onto the $x$-axis parallel to $l(z, \theta(z))$ is an interval, say $(a, b)$. Let $V=I \cap(a, b) \cap U$. It is readily verified that if $x \in A_{m k} \cap V$, then $S_{x r} \cap S_{z r} \neq \varnothing$.

Suppose that $\left\{x_{i}\right\}$ is any sequence of points in $Q$ with $z$ as limit. Then there is an $N>0$ such that $i>N$ implies $x_{i} \in V$. Let $i>N$ be fixed and let $n\left(x_{i}\right)$ be the smallest integer $n$ such that $x_{i} \in A_{n}$. For any $x \in Q \cap V$ we have that $\mid \theta(x)$ $-\theta\left(x_{i}\right) \mid<1 / 2 k$, and hence for $x \in A_{m k} \cap V$ we have $\alpha(x) \geqq \theta(x)+1 / k \geqq \theta\left(x_{i}\right)$ $+1 / 2 k$ and $\beta(x) \leqq \theta(x)-1 / k \leqq \theta\left(x_{i}\right)-1 / 2 k$. From the fact that $A_{m k}$ is dense in $Q \cap V$ and from the previous statement, it follows that there is $w \in A_{m k} \cap V$ for which $S_{w m} \cap S_{x_{i} n\left(x_{i}\right)} \neq \varnothing$. Also, $S_{w m} \cap S_{z n(z)} \neq \varnothing$.

The proof is completed in the same way as in Theorem 1.

Next we consider boundary functions $f$ which are obtained by taking the limit of the function $\Phi$ of two variables along two simple arcs with endpoint $(x, 0)$, the arcs being "separated" by a line $l(x, \theta)$. First, we give an example which shows that we cannot expect to get as "good" a result in this case as in Theorem 2.

EXAMPLE 2. Let $\bigcup_{n=1}^{\infty}\left(a_{n}, b_{n}\right)$ be the complement relative to $[0,1]$ of the Cantor set in $[0,1]$. For each $n=1,2, \cdots$, construct an isosceles triangle in the upper half-plane with $\left[a_{n}, b_{n}\right]$ as base and with the angles at " $a_{n}$ " and " $b_{n}$ " equal to $\left(\pi / 2-1 / 2^{n}\right)$. Then let $T_{n}$ be the set of all points $(u, v)$ inside or on the triangle with base $\left[a_{n}, b_{n}\right]$ for which $0<v \leqq 1 / n$. Define $\Phi: R \times R^{+} \rightarrow R$ as follows:

$$
\Phi(u, v)=\left\{\begin{array}{l}
0 \text { if }(u, v) \in \bigcup_{n=1}^{\infty} T_{n}, \\
1 \text { if }(u, v) \notin \bigcup_{n=1}^{\infty} T_{n} .
\end{array}\right.
$$

The "angle" function $\theta: R \rightarrow(0, \pi)$ is defined to be equal to $\pi / 2$ on

$$
R \sim \bigcup_{n=1}^{\infty}\left[a_{n}, b_{n}\right],
$$

equal to $\pi / 2-1 / 2^{n-1}$ at $x=a_{n}$, and equal to $\pi / 2+1 / 2^{n-1}$ at $x=b_{n}$ and is defined linearly between each $a_{n}$ and $b_{n}$. Then $\theta$ is in the first Baire class.

For each $x$ in $R$ we select two simple $\operatorname{arcs} \Gamma_{x}^{1}$ and $\Gamma_{x}^{2}$, each with $(x, 0)$ as an endpoint, which except for the point $(x, 0)$ lie in the upper half-plane and on opposite sides of the line $l(x, \theta(x))$. For $x=a_{n}$ we further require $\Gamma_{x}^{1}$ to be the side of $T_{n}$ and $\Gamma^{2}$ to lie inside $T_{n}$ to the right of $l(x, \theta(x))$. Similarly, for $x=b_{n}$ we require $\Gamma_{x}^{2}$ to be the side of $T_{n}$ and $\Gamma_{x}^{1}$ to lie inside $T_{n}$ to the left of $l(x, \theta(x))$. Also for 
points $x$ in $[0,1] \sim \bigcup_{n=1}^{\infty}\left[a_{n}, b_{n}\right], \Gamma_{x}^{1}$ and $\Gamma_{x}^{2}$ are chosen so as to avoid $\bigcup_{n=1}^{k+1} T_{n}$ as they pass from the line $y=1 / k$ to the line $y=1 /(k+1)$.

It is clear that the boundary limit of $\Phi$ relative to the simple $\operatorname{arcs} \Gamma_{x}^{1}$ and $\Gamma_{x}^{2}$ exists for each $x$, and the boundary function $f$ thus obtained is equal to zero on $\bigcup_{n=1}^{\infty}\left[a_{n}, b_{n}\right]$ and is equal to one on the complement of $\bigcup_{n=1}^{\infty}\left[a_{n}, b_{n}\right]$. As we have observed before, this function $f$ is not in the first Baire class.

Definition. Let $\mathscr{C}$ be a family of simple arcs lying in the upper half-plane except that each has an end point on the $x$-axis. Suppose also that for each $x$ in $R$ there are exactly two arcs having $(x, 0)$ as an end point and these two arcs are separated by a line $l(x, \theta(x))$ where $\theta: R \rightarrow(0, \pi)$ is some continuous function. Then $\mathscr{C}$ is said to be continuously directed.

THEOREM 3. If $f$ is a boundary function of a function $\Phi: R \times R^{+} \rightarrow R$ obtained by taking limits of $\Phi$ relative to a continuously directed family $\mathscr{C}$ of simple arcs, then $f$ is in the first Baire class.

Proof. Let $\theta: R \rightarrow(0, \pi)$ be a continuous function which "directs" the arcs and for each $x$, let $\Gamma_{x}^{1}$ denote the arc lying to the left of $l(x, \theta(x))$ and $\Gamma_{x}^{2}$ the other arc. Also, let $\Gamma_{x}$ denote the union of $\Gamma_{x}^{1}$ and $\Gamma_{x}^{2}$.

The first part of the proof proceeds as the proof of Theorem 1 except that $\Gamma_{x}$ plays the role of $S_{x}$ and $\Gamma_{x n}$ is the set of points $(u, v)$ in $\Gamma_{x}$ for which $0<v \leqq 1 / n$. Then let $D_{n}, D_{n_{0}}, Q, M, A_{n}, k$, and $I$ be as in the proof of Theorem 1 .

Let $z$ be a fixed point in $Q \cap I$, and let $n(z)$ be the smallest positive integer $n$ such that $z \in A_{n}$. We assert that there is an open interval $V \subset I$ with $z \in V$ such that $\Gamma_{x_{j}} \cap \Gamma_{z j} \neq \varnothing$ for $j=\max \{n(z), k\}$ and any $x \in A_{k} \cap V$. Moving from $(z, 0)$ along $\Gamma_{z}^{1}$ let $p_{1}$ be the first point which lies on the line $y=1 / j$. Similarly, let $p_{2}$ be the first point on $\Gamma_{z}^{2}$ which lies on $y=1 / j$. There are numbers $\alpha$ and $\beta$ between 0 and $\pi$ such that $p_{1}$ lies on $l(z, \alpha)$ and $P_{2}$ lies on $l(z, \beta)$. Set $\varepsilon$ equal to $2^{-1}$ $\cdot \min \{\alpha-\theta(z), \theta(z)-\beta\}$. Since $\theta$ is continuous and $\varepsilon$ is positive, there is an open interval $U$ containing $z$ such that if $x \in U$, then $|\theta(z)-\theta(x)|<\varepsilon$. Let $(a, b)$ be the interval obtained by projecting $p_{1}$ in a direction parallel to $l(z, \alpha-\varepsilon)$ onto the $x$-axis and by projecting $p_{2}$ in a direction parallel to $l(z, \beta+\varepsilon)$ onto the $x$-axis. Let $V=U \cap(a, b)$. If $x$ is in $A_{k} \cap V$ and $x<z$, then the arc at $x$ which lies to the right of $l(x, \theta(x))$ must meet $\Gamma_{z}^{1}$ below the line $y=1 / j$. Likewise, if $x$ is in $A_{k} \cap V$ and $x>z$, then the arc at $x$ which lies to the left of $l(x, \theta(x))$ must meet $\Gamma_{z}^{2}$ below the line $y=1 / j$.

Let $\left\{x_{i}\right\}$ be any sequence of points in $Q$ with $z$ as limit. There is a positive number $N$ such that $i>N$ implies $x_{i} \in V$. Let $k>N$ be fixed, and let $n\left(x_{i}\right)$ be the smallest positive integer $n$ such that $x_{i} \in A_{n}$. Since $A_{k}$ is dense in $V$ and $\theta$ is continuous, there is an $x^{*}$ in $A_{k} \cap V$ such that $\Gamma_{x^{*} k} \cap \Gamma_{x_{i} n\left(x_{i}\right)} \neq \varnothing$ (this is verified by using the same method as in the previous paragraph).

The proof is now completed as in the proof of Theorem 1. 
Definition. Two simple arcs $\Gamma_{x}^{1}$ and $\Gamma_{x}^{2}$ (which lie in the upper half-plane except that each has $(x, 0)$ as an end-point) are Stolz separated if there is a Stolz angle $S_{x}$ with vertex $(x, 0)$ which lies between $\Gamma_{x}^{1}$ and $\Gamma_{x}^{2}$ except for the point $(x, 0)$.

THEOREM 4. If $f$ is a boundary function of $\Phi$ obtained by taking the limit of $\Phi$ relative to two Stolz separated arcs at each point $(x, 0)$ on the real line, and if the family of Stolz angles which separate the arcs is B-directed, then $f$ is in the first Baire class.

The proof is like the proof of Theorem 2 except for a few obvious modifications.

4. Arbitrary Stolz angles. In $\$ 2$ an example was given which showed that if a real-valued function $f$ of one variable is a Stolz angle limit of a function of two variables, without any restriction on the Stolz angles, then the function $f$ may not be in the first Baire class. However, the function $f$ in the example was in the second Baire class, and $f$ was equal to a Baire one function except on a countable set. Bagemihl and Piranian [1] refer to such functions as honorary functions of the first Baire class. We will show that any function which is a Stolz angle limit of a function of two variables is an honorary function of the first Baire class.

We now refer to some results due to G. Lederer [5] which will be used in the proof of the next theorem.

Let $\mathscr{N}$ be a countably additive hereditary class of sets. A proposition will be said to hold a.e. in a set $E$ if the subset of $E$ where the proposition fails to hold is a member of $\mathscr{N}$.

Let $\alpha$ be an ordinal number, $\alpha \geqq 1$.

Definition. A function $f$ is said to have the property $D(\alpha)$ with respect to a closed set $F$ at a point $x$ in $F$ if and only if for any positive $\varepsilon$ there is a neighborhood $G$ of $x$ and a function $g$ in the Baire class $\alpha$ such that $|f-g|<\varepsilon$ a.e. in $G \cap F$.

Definition. A function $f$ is said to have the property $\Delta(\alpha)$ if and only if for any nonempty closed set $F$ there is an $x$ in $F$ such that $f$ has the property $D(\alpha)$ with respect to $F$ at $x$.

Lederer has shown that if a function $f: R \rightarrow R$ has the property $\Delta(\alpha)$, then the function is equal a.e. in $R$ to a function in the Baire class $\alpha$.

Notation. If $P$ is a nonempty perfect subset of the real line, we will denote by $\dot{P}$ the set consisting of $P$ minus the end-points of the complementary intervals.

LeMMA. If $f: R \rightarrow R$ is a Stolz angle limit function for $\Phi: R \times R^{+} \rightarrow R$, then $f \mid \dot{P}$ has a point of continuity for any nonempty perfect set $P$.

Proof. Suppose there is a nonempty perfect set $P$ for which $f \mid \dot{P}$ has no point of continuity. Let $D_{n}=\{x \in \dot{P}: \omega(f \mid \dot{P}, x) \geqq 1 / n\}$. Then $\dot{P}=\bigcup_{n=1}^{\infty} D_{n}$ and $P=\bigcup_{n=1}^{\infty} D_{n} \cup(P \sim \dot{P})$. Since $P \sim \dot{P}$ is countable and $P$ is of the second category in itself, there is an integer $n$ and an open interval $I$ such that $I \cap P \neq \varnothing$ and $D_{n_{0}}$ is dense in $I \cap P$. Since $D_{n_{0}}$ is closed relative to $P$, we have that $D_{n_{0}} \supset I \cap P$. We will denote $I \cap P$ by $Q$. 
Let $A_{n}=\left\{x \in Q: p \in S_{x n}\right.$ implies $\left.|\Phi(p)-f(x)|<1 / M\right\}$, where $M$ is a fixed number greater than $16 n_{0}$. Since $\Phi$ converges at each $x$ relative to the set $S_{x}$, we have $Q=\bigcup_{n=1}^{\infty} A_{n}$. Thus there is an $A_{k}$ which is second category in $P$ since $Q$ is of the second category in $P$.

For each rational number $r$ between 0 and $\pi$, let $B_{r}$ be the set of points $x$ in $A_{k}$ for which $l(x, r)$ meets the interior of $S_{x}$. It follows that $A_{k}=\bigcup_{r} B_{r}$. Hence there is a rational number $q$ such that $B_{q}$ is of the second category in $P$, and so there is an open interval $J$ contained in $I$ for which $J \cap P \neq \varnothing$ and $B_{q}$ is dense in $J \cap P$.

Let $z$ be a fixed point in $B_{q} \cap J$. Project $S_{z k}$ to the $x$-axis parallel to the line $l(z, q)$, and denote by $(a, b)$ the interval determined by this projection. The interval $(a, b)$ contains $z$, and also $S_{x k} \cap S_{z k} \neq \varnothing$ for any $x$ in $B_{q} \cap J \cap(a, b)$.

Let $\left\{x_{i}\right\}$ be any sequence of points in $P \cap J$ with $z$ as limit. There is a number $N$ such that $i>N$ implies $x_{i} \in(a, b) \cap J$. Let $i$ greater than $N$ be fixed. Since $x_{i}$ is a bilateral limit point of $B_{q}$, there is an $x^{*}$ in $B_{q} \cap(a, b) \cap J$ for which

$$
S_{x^{*} k} \cap S_{x_{i} n\left(x_{i}\right)} \neq \varnothing,
$$

where $n\left(x_{i}\right)$ is the smallest positive integer $n$ for which $x_{i} \in A_{n}$. Let $\xi$ be a point in $S_{z k} \cap S_{x^{*} k}$ and let $\eta$ be a point in $S_{x k} \cap S_{x_{i} n\left(x_{i}\right)}$. It follows that

$$
\begin{aligned}
\left|f(z)-f\left(x_{i}\right)\right| \leqq & |f(z)-\Phi(\xi)|+\left|\Phi(\xi)-f\left(x^{*}\right)\right| \\
& +\left|f\left(x^{*}\right)-\Phi(\eta)\right|+\left|\Phi(\eta)-f\left(x_{i}\right)\right| \leqq \frac{4}{M}<\frac{1}{4 n_{0}} .
\end{aligned}
$$

This implies that $\omega(f \mid \dot{P}, z) \leqq 1 / 2 n_{0}$, which is a contradiction. Therefore $f \mid \dot{P}$ has a point of continuity.

THEOREM 5. If $f: R \rightarrow R$ is a Stolz angle limit function for $\Phi: R \times R^{+} \rightarrow R$, then $f$ is an honorary function of the first Baire class.

Proof. We will show that $f$ has the property $\Delta(1)$ for $\mathscr{N}$ being the class of all countable subsets of $R$. Thus from Lederer's theorem we conclude that $f$ is equal to a function in Baire class one except on a countable set.

Let $F$ be any nonempty closed subset of $R$. If $F$ has an isolated point $x$, then trivially $f$ has $D(1)$ with respect to $F$ at $x$.

Let us assume $F$ is a perfect set. Then $f \mid \dot{F}$ has a point of continuity, say $x_{0}$. Let $\varepsilon>0$ be given. There is a neighborhood $G$ of $x_{0}$ such that for any $x$ in $G \cap F,\left|f(x)-f\left(x_{0}\right)\right|<\varepsilon$. Let $g(x)=f\left(x_{0}\right)$. Then $|g-f|<\varepsilon$ on $G \cap F$ except for a countable set. Therefore $f$ has the property $D(1)$ with respect to $F$ at $x_{0}$ in $F$, and so $f$ has property $\Delta(1)$ and the proof is complete.

From the lemma preceding Theorem 5 it is easily seen that not all honorary functions of the first Baire class are arbitrary Stolz angle limit functions. For example, the function $f$ which is 1 on the rationals and 0 on the irrationals is an honorary function of the first Baire class. However, this function restricted to 
the open interval $(0,1)$ has no points of continuity and so fails to satisfy the condition that $f \mid \dot{P}$ has a point of continuity for any nonempty perfect set $P$.

Theorem 5 remains true if Stolz separated arcs are used in place of the Stolz angles as the sets on which the limits are taken. The proof involves only a few modifications of the proof of Theorem 5 .

5. Continuous Stolz extensions. In this section we let $[\xi]$ denote the $n$-tuple $\left(\xi_{1}, \cdots, \xi_{n}\right)$ of real numbers and let $\mathscr{C}$ denote the complex numbers. We use superscripts to index sequences of points in $R^{n}$.

Theorem 6 is an improvement of a theorem due to Jarnik [4]. Except for the continuity assertion and the functions being complex-valued, his theorem is the same as Theorem 6.

Let $I$ be a compact interval in $R^{n}$. Then $\Delta$ will denote the subset of $I \times I$ consisting of those points of the form $([\xi],[\xi])$.

THEOREM 6. If $f: I \rightarrow \mathscr{C}$ is a function in the first Baire class, then there is a continuous function $\Phi:(I \times I \sim \Delta) \rightarrow \mathscr{C}$ such that $\lim _{k \rightarrow \infty} \Phi\left([\xi]^{k},[\eta]^{k}\right)=f([x])$ whenever (a) $\lim _{k \rightarrow \infty}[\xi]^{k}=\lim _{k \rightarrow \infty}[\eta]^{k}=[x]$ and (b) $\left(\xi_{i}{ }^{k}-x_{i}\right)\left(\eta_{i}{ }^{k}-x_{i}\right) \leqq 0$ for each $i=1, \cdots, n$ and $k=1,2, \cdots$.

Proof. Let $\left\{f_{m}\right\}$ be a sequence of continuous functions on $I$ for which $\lim _{n \rightarrow \infty} f_{m}([x])=f([x])$ for every $[x]$ in $I$. Define $H: I \times(0,1] \rightarrow \mathscr{C}$ by letting $H([x], 1 / m)=f_{m}[(x)]$ and extending $H$ linearly, that is, for $1 /(m+1)<y \leqq 1 / m$, $H([x], y)=G(y) \cdot f_{m+1}([x)]+(1-G(y)) \cdot f_{m}([x])$, where

$$
G(y)=\left(\frac{1}{m}-y\right) /\left(\frac{1}{m}-\frac{1}{(m+1)}\right) .
$$

Then $H$ is continuous on $I \times(0,1]$, and $\lim _{y \rightarrow 0^{+}} H([x], y)=f([x])$.

Since $I$ is compact, each $f_{m}$ is uniformly continuous. For each $m$ and $\varepsilon>0$, let $\delta_{m}(\varepsilon)$ be a positive number such that $\left|f_{m}([x])-f_{m}([y])\right|<\varepsilon$ whenever the distance from $[x]$ to $[y]$ is less than $\delta_{m}(\varepsilon)$, and set $\zeta_{1}=\min \left\{\delta_{1}(1), \delta_{2}(1), 1\right\}$. Inductively, define $\zeta_{m}=\min \left\{\delta_{m}(1 / m), \delta_{m+1}(1 / m),(m-1) / 2\right\}$ for $m=2,3, \cdots$. The sequence $\left\{\xi_{m}\right\}$ converges monotonically to zero. Next, define $\tau: R^{+} \rightarrow(0,1]$ by letting $\tau(r)=1$, if $r \geqq \zeta_{1}$ and letting $\tau\left(\zeta_{k}\right)=1 / k$ and by extending linearly. Then the function $\theta:(I \times I \sim \Delta) \rightarrow(0,1]$ defined by $\theta=\tau \circ \rho$, where $\rho$ is the usual metric on $R^{n}$, is continuous.

For $([\xi],[\eta]) \in(I \times I \sim \Delta)$, define $\Phi([\xi],[\eta])=H(([\xi]+[\eta]) / 2, \theta([\xi],[\eta]))$. The function $\Phi$ is continuous and, as we will now show, possesses the desired property.

Let $[\xi]^{k}$ and $[\eta]^{k}$ satisfy (a) and (b) of the theorem for some $[x]$. Since $\left(\xi_{i}^{k}-x_{i}\right) \cdot\left(x_{i}-\eta_{i}^{k}\right) \geqq 0$, we have

$$
\rho^{2}\left([\xi]^{k},[\eta]^{k}\right) \geqq \sum_{i=1}^{n}\left[\left(\xi_{i}^{k}-x_{i}\right)^{2}+\left(\eta_{i}^{k}-x_{i}\right)^{2}\right]
$$


and

$$
\rho^{2}\left(\frac{[\xi]^{k}+[\eta]^{k}}{2},[x]\right) \leqq \sum_{i=1}^{n}\left[\frac{\left(\xi_{i}^{k}-x_{i}\right)^{2}}{4}+\frac{\left(\eta_{i}^{k}-x_{i}\right)^{2}}{4}\right]
$$

Thus

$$
\rho\left(\frac{[\xi]^{k}+[\eta]^{k}}{2},[x]\right) \leqq \frac{1}{2} \rho\left([\xi]^{k},[\eta]^{k}\right) .
$$

Let $\varepsilon$ greater than zero be given. There is a positive integer $M$ such that $1 / M<\varepsilon / 2$ and a positive integer $K$ such that $k>K$ implies $\rho\left([\xi]^{k},[\eta]^{k}\right) \leqq \zeta_{M}$. Hence $\theta\left([\xi]^{k},[\eta]^{k}\right) \leqq 1 / M$ for $k>K$. From the definition of the sequence $\left\{\zeta_{m}\right\}$ and that of $H$ it follows, in view of (1), that for $k>K$,

$$
\left|H\left(\frac{[\xi]^{k}+[\eta]^{k}}{2}, \theta\left([\xi]^{k},[\eta]^{k}\right)\right)-H\left([x], \theta\left([\xi]^{k},[\eta]^{k}\right)\right)\right|<\frac{1}{M} .
$$

Since $\lim _{k \rightarrow \infty} \theta\left([\xi]^{k},[\eta]^{k}\right)=0$, it follows from the definition of $H$ that $\lim _{k \rightarrow \infty} H\left([x], \theta\left[(\xi]^{k},[\eta]^{k}\right)\right)=f([x])$. Thus there is a positive integer $K^{\prime}$ such that $k>K^{\prime}$ implies

$$
\left|H\left([x], \theta\left([\xi]^{k},[\eta]^{k}\right)\right)-f([x])\right|<\frac{1}{M} .
$$

Let $K^{\prime \prime}=\max \left\{K, K^{\prime}\right\}$. Then, if $k>K^{\prime \prime}$, both (2) and (3) hold, which together with

$$
\begin{aligned}
& \left|\Phi\left([\xi]^{k},[\eta]^{k}\right)-f([x])\right| \\
& \quad \leqq\left|H\left(\frac{|\xi|^{k}+|\eta|^{k}}{2}, \theta\left([\xi]^{k},[\eta]^{k}\right)\right)-H\left([x], \theta\left([\xi]^{k},[\eta]^{k}\right)\right)\right|
\end{aligned}
$$

imply $\left|\Phi\left([\xi]^{k},[\eta]^{k}\right)-f([x])\right|<1 / M+1 / M<\varepsilon$. This completes the proof.

We now consider the 1-dimensional case of this theorem. Suppose that $f: I \rightarrow \mathscr{C}, I=[a, b]$, is in the first Baire class. According to Theorem 6 , there is a continuous function $\Phi:(I \times I \sim \Delta) \rightarrow \mathscr{C}$ with the property that $\lim _{k \rightarrow \infty} \Phi\left(\xi^{k}, \eta^{k}\right)$ $=f(x)$ for any sequence $\left\{\left(\xi^{k}, \eta^{k}\right)\right\}$ of points converging to $(x, x)$ such that each $\left(\xi^{k}, \eta^{k}\right)$ lies either in the sector to the right of the line $\xi=x$ and below the line $\eta=x$ or in the sector to the left of the line $\xi=x$ and above the line $\eta=x$. In this section we will use the term Stolz angle to refer to angular sectors in $(I \times I \sim \Delta)$ with their vertices on $\Delta$, and we will show that $\Phi$ converges to $f$ for any such Stolz angles.

CoROllary 1. If $f: I \rightarrow \mathscr{C}, I=[a, b]$, is in the first Baire class, then there exists a continuous function $\Phi:(I \times I \sim \Delta) \rightarrow \mathscr{C}$ such that, for each $x, \lim \Phi(\xi, \eta)$ $=f(x)$ as $(\xi, \eta) \rightarrow(x, x)$ relative to any Stolz angle with vertex $(x, x)$. 
Proof. Let $\Phi:(I \times I \sim \Delta) \rightarrow \mathscr{C}$ be the continuous function constructed in the proof of Theorem 6 , and let $(x, x)$ be any point on $\Delta$. In order to prove the corollary, it suffices to show that, for any positive integer $N, \Phi$ converges to $f(x)$ relative to the Stolz angle $S_{N}$ lying below the line $\eta=\xi$ whose sides lie on the lines through $(x, x)$ with slopes $(N+1) / N$ and $N /(N+1)$.

Let $N$ be given, and let $\left(\xi_{k}, \eta_{k}\right)$ be any sequence of points, lying in the Stolz angle described above, with limit $(x, x)$. First, let us consider the case where $\xi_{k}>x, \eta_{k}>x$ for every $k=1,2, \cdots$. Let $W_{k}=\xi_{k}-\eta_{k}$, and associate with $\left(\xi_{k}, \eta_{k}\right)$ the point $\left(x+W_{k}, x\right)$. Since the points $\left(\xi_{k}, \eta_{k}\right)$ lie below the line $\eta=\xi$, we have $\left(\eta_{k}-x\right) /\left(\xi_{k}-x\right) \leqq N /(N+1)$. Thus $\eta_{k}-x \leqq N \cdot W_{k}$.

Let $\left\{\zeta_{m}\right\}$ be the sequence defined as in the proof of Theorem 6. Since $W_{k} \rightarrow 0$, we may assume $W_{k} \leqq \zeta_{1}$ for each $k$. Then define $m(k)$ to be the largest integer $m$ for which $W_{k} \leqq \zeta_{m}$. Since $W_{k} \rightarrow 0$, we have that $m(k) \rightarrow \infty$.

Also, let $\theta$ be defined as in the proof of Theorem 6 , and note that $\theta\left(\xi_{k}, \eta_{k}\right)$ $=\theta\left(x+W_{k}, x\right)$. Since $0 \leqq\left(\xi_{k}+\eta_{k}\right) / 2-\left(x+W_{k}+x\right) / 2 \leqq N \cdot W_{k}$ and $\theta\left(\xi_{k}, \eta_{k}\right)$ $\leqq 1 / m(k)$, we have $\left|\Phi\left(\xi_{k}, \eta_{k}\right)-\Phi\left(x+W_{k}, x\right)\right|=\mid H\left(\left(\xi_{k}+\eta_{k}\right) / 2, \quad \theta\left(\xi_{k}, \eta_{k}\right)\right)$ $-H\left(\left(x+W_{k}+x\right) / 2, \theta\left(\xi_{k}, \eta_{k}\right)\right) \mid \leqq N \cdot 1 / m(k)$. Now it is easily seen that the sequence $\left(x+W_{k}, x\right)$ satisfies the conditions (a) and (b) of Theorem 6. Therefore $\lim _{k \rightarrow \infty} \Phi\left(x+W_{k}, x\right)=f(x)$, and so $\lim _{k \rightarrow \infty} \Phi\left(\xi_{k}, \eta_{k}\right)=f(x)$.

The case where $\xi_{k}<x, \eta_{k}<x$ (for every $k=1,2, \cdots$ ) is treated similarly, and the case where $\xi_{k} \geqq x$ and $\eta_{k} \leqq x$ has already been done by Theorem 6 . For an arbitrary sequence, one considers the three subsequences determined by the three cases. For each subsequence $\Phi$ has limit $f(x)$; hence $\Phi$ has limit $f(x)$ relative to the Stolz angle $S_{N}$.

Gleyzal [1] has shown that if a function $f:[a, b] \rightarrow R$ is in thefirst Baire class, then there is a convergent interval function $\phi$ with $f$ as its limit, i.e., there is a function $\phi$ defined on the collection of subintervals of $[a, b]$ such that if $I_{n}$ is a sequence of intervals with lengths going to zero and with $x \in \bigcap I_{n}$, then $\phi\left(I_{n}\right) \rightarrow f(x)$. Indeed, Theorem 6 yields an interval function with this property. For if $\Phi$ is the function given by Theorem 6 , then, for $I \subset[a, b]$, let $\phi(I)=\Phi(u, v)$, where $u$ and $v$ are the left and right end-points, respectively, of $I$. Let $\left\{I_{n}\right\}$ be a sequence of intervals each containing $x \in[a, b]$ such that the lengths converge to zero, and let $\left\{\left(u_{n}, v_{n}\right)\right\}$ be the sequence of points in $[a, b] \times[a, b]$ such that $u_{n}$ and $v_{n}$ are the left and right end-points of $I_{n}$. It is easily checked that this sequence of points satisfies the conditions (a) and (b) of Theorem 6. Hence

$$
\lim _{n \rightarrow \infty} \phi\left(I_{n}\right)=\lim _{n \rightarrow \infty} \Phi\left(u_{n}, v_{n}\right)=f(x) .
$$

In addition, this interval function is "continuous" in the sense that if $I_{0} \subset[a, b]$ and $I$ is another interval in $[a, b]$ such that the measure of the symmetric difference of $I_{0}$ and $I$ is sufficiently small, then the difference $\left|\phi\left(I_{0}\right)-\phi(I)\right|$ is small.

Moreover, Corollary 1 implies that the convergence property of the interval 
function $\phi$ is even stronger. To illustrate this, let us suppose that $\left\{I_{n}\right\}$ is a sequence of intervals lying to the right of $x$ such that (i) given any $\varepsilon>U$, there is an integer $K>0$ such that $n>K$ implies $I_{n} \subset(x, x+\varepsilon)$ and (ii) $\left|I_{n}\right| / d\left(x, I_{n}\right) \geqq 1 / N$ for some integer $N>0$, where $\left|I_{n}\right|$ denotes the length of $I_{n}$ and $d\left(x, I_{n}\right)$ denotes the distance from $x$ to $I_{n}$. Letting $u_{n}$ and $v_{n}$ denote the end-points of $I_{n}$, we have $1 / N \leqq\left|I_{n}\right| / d\left(x, I_{n}\right)=\left(v_{n}-u_{n}\right) /\left(u_{n}-x\right)$. From this we have that $u_{n}-x \leqq N\left(v_{n}-u_{n}\right)$ and $(N+1) \cdot\left(u_{n}-x\right) \leqq N\left(v_{n}-x\right)$. Thus $\left(v_{n}-x\right) /\left(u_{n}-x\right) \geqq(N+1) / N$. Therefore the sequence of points $\left(u_{n}, v_{n}\right)$ in $[a, b] \times[a, b]$ lies in a Stolz angle with vertex $(x, x)$ and converges to $(x, x)$. Hence, by Corollary $1, f(x)=\lim _{n \rightarrow \infty} \Phi\left(u_{n}, v_{n}\right)$ $=\lim _{n \rightarrow \infty} \phi\left(I_{n}\right)$.

Similarly, if $\left\{I_{n}\right\}$ is a sequence of intervals lying to the left of $x$ which get arbitrarily near $x$ as described above and such that $\left|I_{n}\right| / d\left(x, I_{n}\right) \geqq 1 / N$ for some $N>0$, then the sequence $\left\{\left(u_{n}, v_{n}\right)\right\}$ of associated points in $[a, b] \times[a, b]$ lies in a Stolz angle with vertex at $(x, x)$ and the sequence converges to $(x, x)$. Thus again we have by Corollary 1 that

$$
f(x)=\lim _{n \rightarrow \infty} \Phi\left(u_{n}, v_{n}\right)=\lim _{n \rightarrow \infty} \phi\left(I_{n}\right) .
$$

Remark. The above condition that the ratio of $\left|I_{n}\right|$ to $d\left(x, I_{n}\right)$ be bounded away from zero cannot be omitted.

From Corollary 1 it is not difficult to obtain the following slightly different result

COROLlary 2. If $f$ is a complex-valued function of Baire class one on the unit circle, then there is a continuous $F$ defined on the unit disk such that the nontangential limit of $F$ at $e^{i \theta}$ exists and is equal to $f\left(e^{i \theta}\right)$ for each $\theta$.

The author is grateful to the referee for his helpful suggestions for improvements in the paper.

\section{REFERENCES}

1. F. Bagemihl and G. Piranian, Boundary functions for functions defined in a disk, Michigan Math. J. 8 (1961), 201-207.

2. A. Gleyzal, Interval functions, Duke Math. J. 8 (1941), 223-230.

3. F. Hausdorff, Set theory, New York, Translation of 3rd edition (1937) of Mengenlehre, pp. 288-289.

4. V. Jarnik, Sur les fonctions de la premiere classe de Baire, Bull. Internat. Acad. Sci. Boheme (1926).

5. G. Lederer, Two theorems on Baire functions in separable metric spaces, Quart. J. Math. Oxford Ser. 11 (1960), 269-274.

PURdue UnIVersity.

$$
\text { LAFAYETTE, INDIANA }
$$

